This item was submitted to Loughborough's Research Repository by the author.

Items in Figshare are protected by copyright, with all rights reserved, unless otherwise indicated.

\title{
Autonomous recording system for simultaneous long-term ambient noise and marine mammal monitoring
}

PLEASE CITE THE PUBLISHED VERSION

http://dx.doi.org/10.1109/OCEANS.2016.7761467

PUBLISHER

(C) IEEE

VERSION

AM (Accepted Manuscript)

\section{PUBLISHER STATEMENT}

This work is made available according to the conditions of the Creative Commons Attribution-NonCommercialNoDerivatives 4.0 International (CC BY-NC-ND 4.0) licence. Full details of this licence are available at: https://creativecommons.org/licenses/by-nc-nd/4.0/

\section{LICENCE}

CC BY-NC-ND 4.0

\section{REPOSITORY RECORD}

Lepper, Paul A., L. Simon, and L. Dufrechou. 2019. "Autonomous Recording System for Simultaneous Longterm Ambient Noise and Marine Mammal Monitoring". figshare. https://hdl.handle.net/2134/23466. 


\section{Autonomous recording system for simultaneous long-term ambient noise and marine mammal monitoring}

\author{
P.A. Lepper \\ Loughborough University \\ Loughborough, UK \\ p.a.lepper@lboro.ac.uk
}

\author{
L.Simon \& L.Dufrechou \\ RTSYS Ltd \\ Caudan, France \\ 1simon@rtsys.fr \& ldufrechou@rtsys.fr
}

\begin{abstract}
There has be significant growth in recent year in the requirement for high quality long-term underwater acoustic data acquisition. One of the primary drivers has been the assessment of long-term trends in noise in our oceans and potential impacts from anthropogenic noise on marine wildlife. These recorders usually fulfill a number of key roles in this sector. These include providing acoustic data on long-term trends in ambient or background noise, data on trends in system or device noise of interest and data on vocalizing marine species and potential associated behaviors. Many marine sectors now routinely use these data types to assess any impact from their operations and commonly they form part of ocean operators consenting processes. However no single technology is widely available to perform all of these functions efficiently within a single deployment package.
\end{abstract}

Acquisition of high quality data offers a number of major challenges commonly seen in the diversity of requirements listed above. These include operation across wide dynamic ranges and wide bandwidths (a few $\mathrm{Hz}$ - many hundreds of $\mathrm{kHz}$ ) with low self-noise for long periods of time (months to years) in a standalone low-footprint recorder package. A common approach to extend acquisition periods is to use recording duty-cycle to sample for example ambient and device noise regularly on a fixed schedule. This approach however is less useful for capturing uncorrelated biological acoustic events for example echolocating marine mammals in combination with analyzing long-term noise trends.

A dual processing approach combined in an autonomous acoustic data logger is presented. The development of custom embedded low-power electronics has allowed the development of integrated systems combining real-time processing and storage of ambient noise raw data as well as the extraction key acoustic indicators. In parallel a continuous $500 \mathrm{kHz}$ sampling data system is integrated with various frequency/level detectors for detecting high frequency vocalizations of marine mammals and the storage of both detection parameters and full bandwidth raw data of the signal. Work presented in this paper represent technology development to provide key attributes of these requirements in a single continuously sampling unit.

Keywords-Cetacean logger, broadband, continuous monitoring, harbor porpoise, noise assessment, autonomous recorder

\section{Methodology}

\section{A. System Overview}

A dual approach has been taken to overcome the often conflicting requirments of wide bandwidths, varying event occourance and continious monitoring. The developed system arcitecture allows continious sampling across a full bandwith from a few Hertz to several hundred kilo-Hertz .

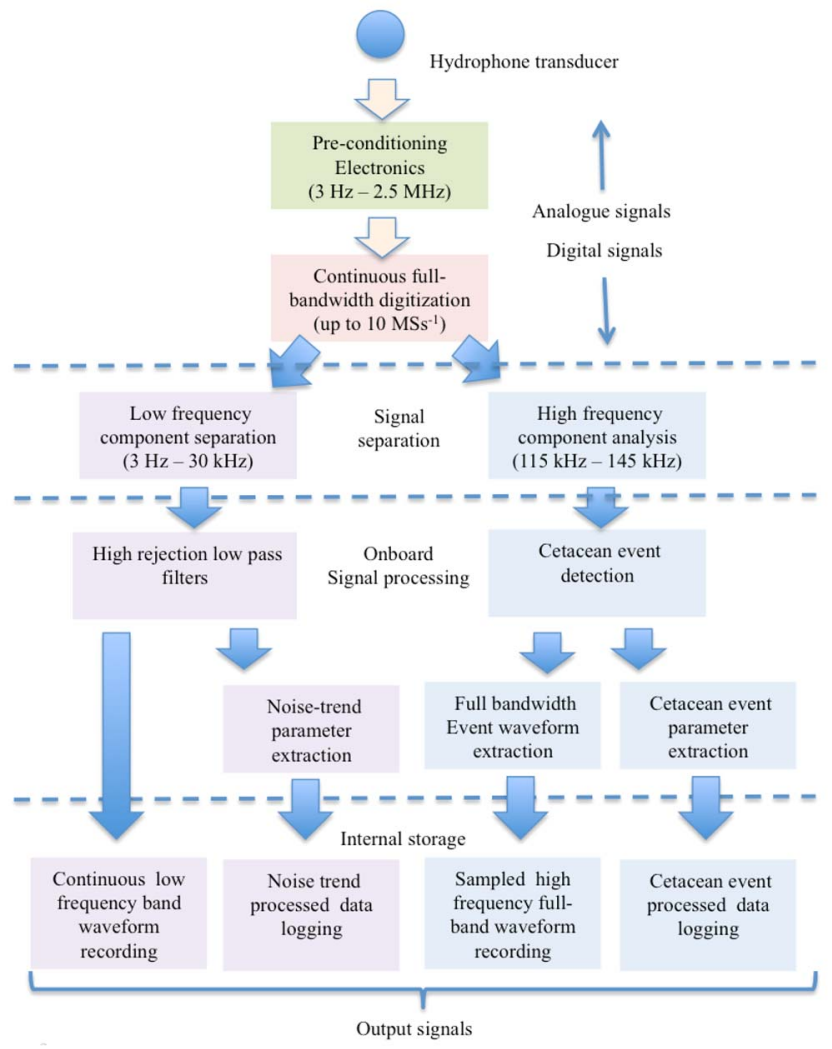

Fig. 1. Overview of system arcitecture

Data is then split into two bands, a lower frequency band $10 \mathrm{~Hz}-30 \mathrm{kHz}$. These data can be directly recorded but also key acoustic parameters exstracted in real-time and stored and / or transitted. High frequency signals typically in the band grater than $100 \mathrm{kHz}$ are also monitored for the presence of cetacean signals. Once detected these signals are also paramrerized and this data stored. 


\section{B. SDA14 Platform}

To implement the proposed methodology the capabilities of SDA14 broadband recorder system from RTSys has been developed to allow real-time signal processing. The SDA14 is a low consumption compact OEM recording platform with directly connectable to transducers, that can acquire very wideband 24-bits signal $\left(10 \mathrm{MSs}^{-1}\right)$ across 4 analog channels with large dynamic range. The platform is also equipped with an embedded DSP (3 GFlops) which can be used for the integration of real time data analysis such as pulse compression, fixed point and floating point computation as well as acoustic feature extraction. The OEM platform is fully programmable thanks to ARM processor running Linux. Data can be stored on different embedded storage media - up to 2TB. The full system architecture is shown in Fig. 2.

The system runs autonomously with independent 8-26V DC power supply and directly programmable but with the capability to be integrated with other instruments and platform through Ethernet or serial port interfaces. The Ethernet port provides general use features such as the programing/data gathering and in-field test of acoustics. The serial port is dedicated to either send back computed data in real time if connected to a underwater modem and to eventually control the system via a master electronic board whilst submerged.

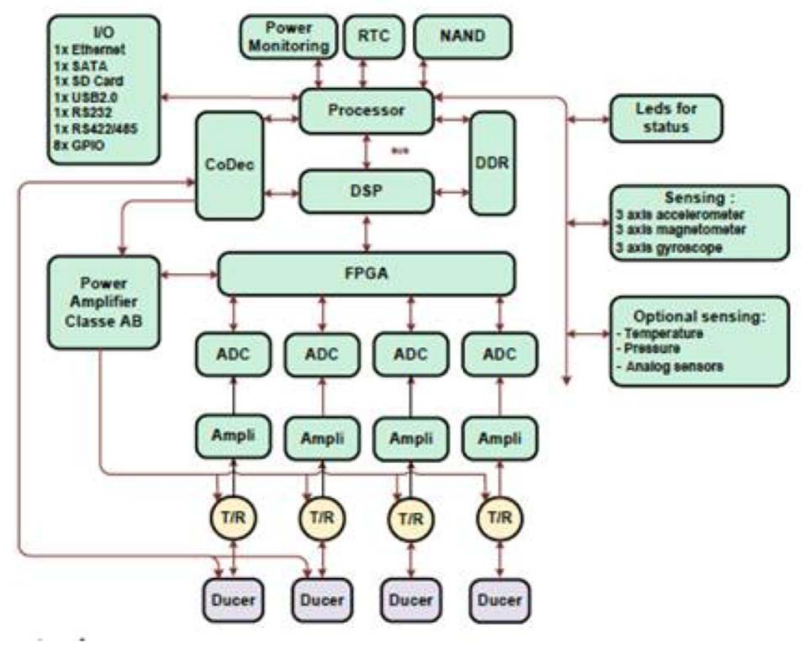

Fig.2. Multi channel SDA14 platform system architecture

In term of energy the board consume $1.6 \mathrm{~W}+0.2 \mathrm{~W}$ per channel while recording and processing and support cycling recording mode (on/off cycles) to adapt the energy consumed. The device can be connected to various transducers types. High-frequency and low noise hydrophones such as GP0190 from COLMAR or TC4032 and TC4014 from RESON have been used.

\section{Cetacean detection algorthym}

1) High frequency cetacean signals
Of primary interest often in Northern European waters is the monitoring of harbor porpoise (Phocoena phocoena). These small cetaceans are widespread echolcators with primary signals between of greater than $115 \mathrm{kHz}$ [1]. These animals have also been shown to be high sensitivity both physiologically [2] and behaviorally [3-4] to anthropogenic noise sources as well as being one of the highest frequency echolocating species. As such these were chosen as primary detection species for the described system development. Fig. 3. Shows an example of a full bandwidth porpoise echolocation 'click' with typical pulse duration to greater 90$110 \mu$ s digitized at a $480 \mathrm{kSs}^{-1}$ sample rate.

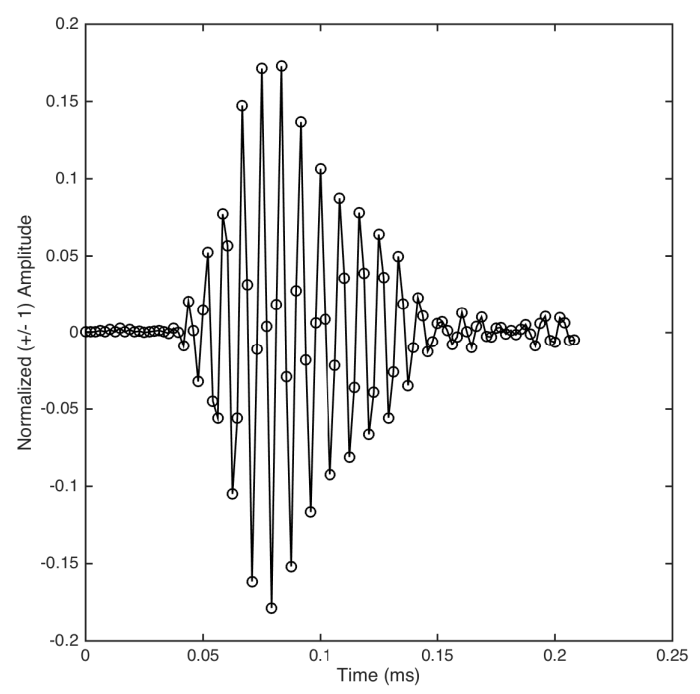

Fig. 3. Example of an on-axis broadband recording of a single harbor porpoise click at a sample rate of $480 \mathrm{kSs}^{-1}$ with good hydrophone sensitivity to above $200 \mathrm{kHz}$.

The primary frequency components are between $115 \mathrm{kHz}$ and $145 \mathrm{kHz}$ [1]. To digitize these bandwidths requires high sample rates and wide band hydrophone systems. In this case requiring a minimum sample rate based on Nyquist-Shannon theorem of around $300 \mathrm{kSs}^{-1}$. The example given shows a reasonable representation of a typical good signal to noise porpoise click at a higher $480 \mathrm{kSs}^{-1}$ sample rate. Data recording at sample rate of up to $1 \mathrm{MSs}^{-1}$ or higher can be achieved through a variety of commercially available systems. These systems have been available for several years however until relatively recently performing real-time analysis, particularly on embedded systems, at these samples rates has been a major challenge due to electronic limitation.

The developed system uses significant real-time processing using a ARM9 core processor to allow continuous real-time analysis using a sample rate of $625 \mathrm{kSs}^{-1}$ to a 24-bit resolution within a fully autonomous system. This was felt sufficient to capture good quality full-bandwidth recordings 
whilst maintaining continuous monitoring and real-time analysis using the SDA14 platform.

\section{2) Cetacean 'click' detector alogorthym.}

A click cetacean click detection process was described by Newborough et al [5] using an envelope detection method. The main process is shown in Fig. 3. This original system was implemented in analogue electronics and field-tested on harbor porpoises and bottlenose dolphins both in captivity and in the wild. Other echolocating species detector were also developed for pilot whales and sperm whales.

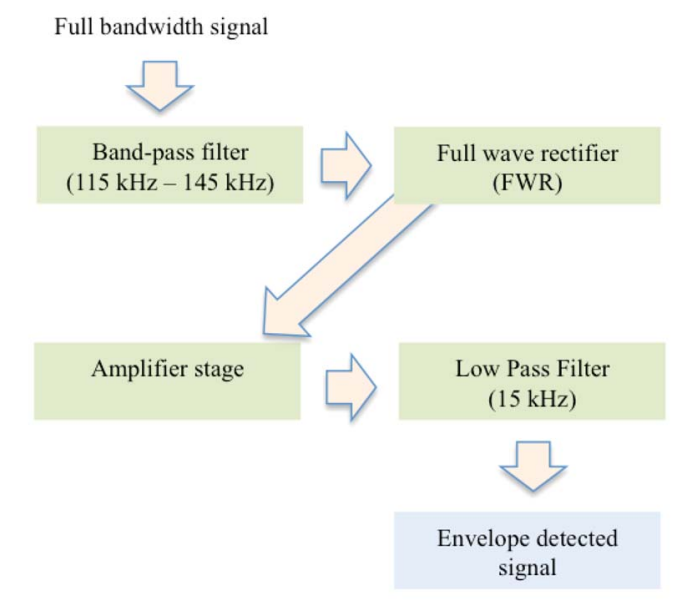

Fig.4 Envelope detection process for cetacean echolocation click processing

From Fig. 4. the broadband signal from a hydrophone is pre-conditioned and then passed through a $4^{\text {th }}$ order band pass Butterworth filter with a $30 \mathrm{kHz}$ bandwidth center frequency $130 \mathrm{kHz}$. This isolates the main click energy from other ocean noise. The signal is then full wave rectified, additional gain controls are then included amplifying the signal until finally passing the signal through a low-pass $2^{\text {nd }}$ order Butterworth filter. This has the action of tracing the envelope of the pulse as shown in Fig. 5. for a short click sequence. The upper panel shows the full bandwidth signal of a harbor porpoise click sequence. The lower panel the processed enveloped detected data. Due to the short duration $(\sim 100 \mu \mathrm{s})$ both the amplitude and timing of the pulse is maintained although the broadband signal waveform is not maintained. This data can then be digitized at much lower bandwidths, less than $20 \mathrm{kHz}$, and is within the hearing range of a human operator giving an impression of the acoustic behavior in real time. Long term continuous signals, such as a tonal even in the $115-145 \mathrm{kHz}$ band, would be eliminated through this process.

The parameter amplitude and the inter-click period between two successive clicks have been used to log and study cetacean acoustic behaviors. Inference from in particular the inter-click periods has been used for interpolation of behaviors such as feeding, navigation, social buzzes. Separation of individual click trains from large groups of dolphin on envelope-detected data was demonstrated by Dumortier et al allowing study of complex group dynamics [6]. Using interclick period changes was used to interpret harbor porpoise behaviors around an active North Sea drilling platform [7], piling operation [8] as well as many others.
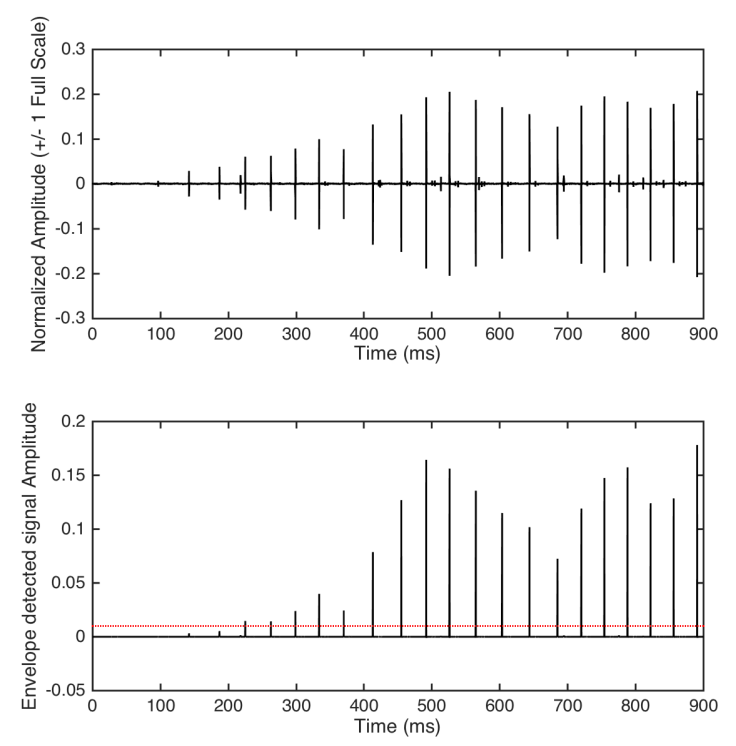

Fig. 5. Upper panel broadband harbor porpoise click sequence. Lower panel processed click signals using the envelope detection method. Red line indicates a simple level detection for click identification.

The current system has taken this original analog electronic design and implemented in real-time Digital Signal Processing using the SDA14 platform architecture. The high samples rates and digital implementation of the current system has also allowed full bandwidth waveform extraction / storage. i.e. a short duration full-bandwidth waveform signal including the whole click signal is buffered and recorded each time a click event is detected. This offers significant advantages in cetacean studies including full spectrum frequency analysis and potential for array processing i.e. waveform phase difference of individual arrivals on multiple spatially distributed hydrophones could be used to localize the source to much higher resolution than available from the envelope processed data alone using a conventional time-ofarrival methods. Systems to do this kind of processing (full waveform / multi-channel) usually require significant processing power and commonly used from towed arrays with surface or cabled connections. The current autonomous implementation of full waveform capture provided potential for long term stand-alone detection and localization of cetacean species as well as continuous background noise assessment.

\section{Low frequency analysis}

Using the on-board processing unit low-frequency parameter analysis is carried out in parallel with the cetacean signal 
analysis as well as continuous low bandwidth recording and selected 'event triggered' high bandwidth recording as described in Fig. 1. For continuous signals processed data includes the fixed period integrated Sound Exposure Level (SEL), Sound Pressure Level (SPL) and zero-to-peak Sound Pressure Level. For transient signals the pulse integrated Sound Exposure Level and Peak Sound Pressure Level are calculated as well at the broadband integrated level and third octave band levels. These parameters form the most common acoustic metrics for assessment of impact of noise on marine wildlife. Real-time processing of these metrics using onboard systems offers significant advantages in saving post processing time and potential for real-time monitoring and feedback via a variety of communication protocols including Radio Frequency (RF) links and underwater modems.

\section{RESULTS}
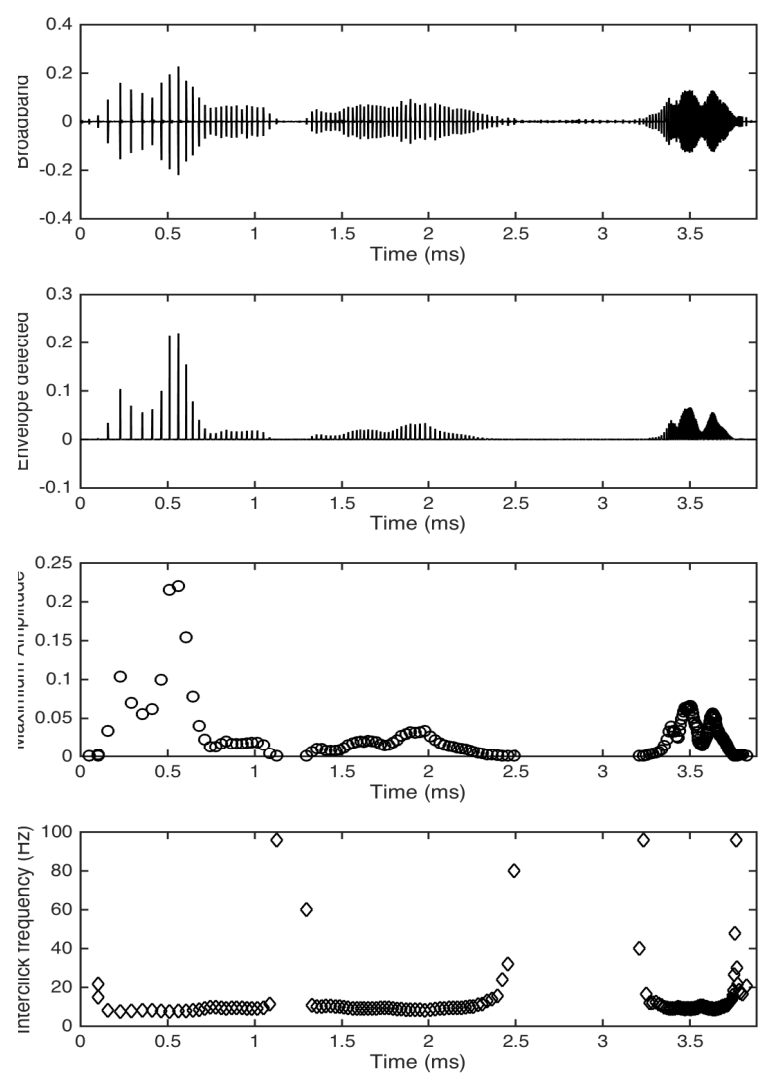

Fig.6. Upper panel is raw broadband waveform files for harbor porpoise echolocation train. Second panel is processed envelope detected data using the propose envelope detection method. The third panel shows the maximum amplitude of each detected pulse or click. The lower panel the inter-click frequency for successive pulses.

Fig. 6. shows graphed processed data using MATLAB for a harbor porpoise echolocation click sequence extracted using the proposed methodology. The upper panel shows the broadband click waveforms for the whole click train. This is followed using the envelope detected waveforms in the second panel. Note that the detection method can work with very poor signal to noise signals due to the band-pass and transient detection of the system. A simple threshold detection is then used in the third panel to identify individual clicks, the maximum per click level is then extract and shown in the third panel and the inter-click period as a frequency between clicks is shown in the last panel. Note the variation in the inter-click period even for relatively small amplitude signal. These data can be extracted in real-time and stored for real-time transmission as well as storage.

In addition individual broadband time waveforms can be captured and stored for post analysis. Fig.7. shows an example of the algorithm captured click signal automatically extracted from the previous shown data set. These data are time stamped and saved independently to the lower band continuous recording data.

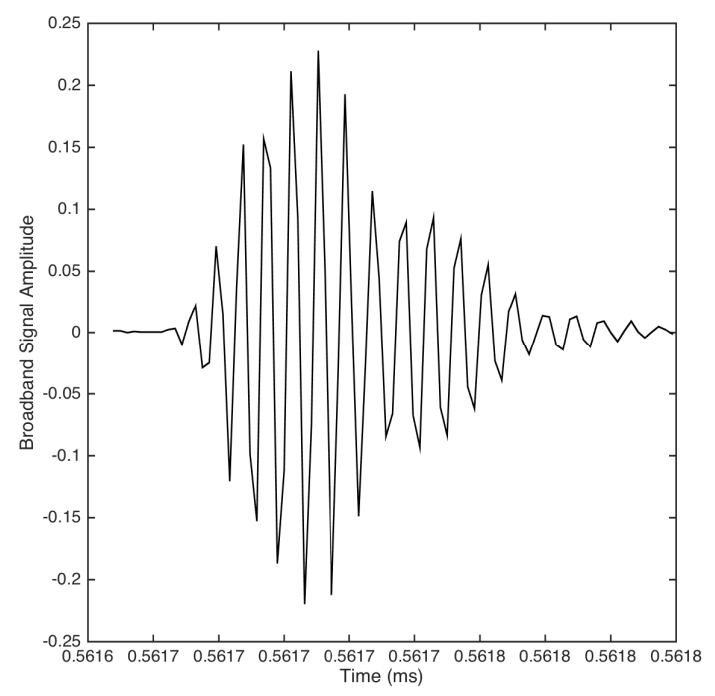

Fig.7. Automated $\sim 500 \mathrm{kSs}^{-1}$ broadband signal extraction and storage using the real-time click detection algorithm

These data combined with continuous lower frequency noise monitoring and parameter capture provides a unique sound sampling capability for long-term autonomous or realtime monitoring deployments.

\section{DISCUSSION}

The developed system offers an advance in real-time data capture and signal processing on a low form-factor autonomous underwater recording system for whole acoustic environment monitoring. This combined with use of radio telemetry or use of underwater modems offers opportunity for real time parameter analysis in the growing demands for a variety of acoustic monitoring requirements. The system itself 
is configurable with a variety of hydrophone transducers. The overall noise performance and bandwidth is therefore ultimately limited by the quality of both the analogue front end and the digital system described above.

The data shown in Fig. 5. and Fig. 6. also illustrate another limitation of the cetacean click detection methodology. Often high amplitude variations are seen across successive clicks due to the high directivity of the animals sonar. This narrow beam signal can result in poor signal to noise ratio on the broadband waveform as the animal move off-axis from the hydrophone. The use of the band pass filter improves this performance particularly in the presence of other noise. In some cases detection of clicks at $0 \mathrm{~dB}$ signal-to-noise ratio can be made in the presence of continuous white noise. Other signal processing methods such as cross-correlation also offer opportunities on the SDA14 platform to improve detection and classification tasks.

The current design is aimed specifically at harbor porpoises. This works well for the porpoise, as their operational band is not widely used by other species. The same process has been carried out for dolphins species using a band-pass filter $60 \mathrm{kHz}-100 \mathrm{kHz}$ and even lower for larger echolocating whales. This scalability of the design offers significant versatility as new species specific algorithms are developed particular combined with whole acoustic environment assessment.

\section{REFERENCES}

[1] W. C. Verboom, and R. A. Kastelein, Acoustic signals by harbour porpoises (Phocoena phocoena), in Harbour Porpoises-Laboratory Studies to Reduce Bycatch, edited by P. E. Nachtigall, J. Lien, W. W. L. $\mathrm{Au}$, and A. J. Read De Spil, Woerden, The Netherlands, 1995, pp. 1-39.

[2] K. Lucke, U. Siebert, P.A. Lepper, M.A. Blanchet, "Temporary shift in masked hearing thresholds in a harbor porpoise (Phocoena phocoena) after exposure to seismic airgun stimuli", J.Acoust.Soc.Am, 125(6), doi: 10.1121/1.3117443, 2009, pp. 4060-70.

[3] M. J. Brandt, A. Diederichs, K. Betke, G. Nehls, "Responses of harbour porpoises to pile driving at the Horns Rev II offshore wind farm in the Danish North Sea", Mar Ecol Prog Ser, Vol. 421, doi: 10.3354/meps08888, 2011 pp. 205-216.

[4] M. Dyndo, D.M. Wiśniewska, L. Rojano-Doñate, P.T. Madsen, "Harbour porpoises react to low levels of high frequency vessel noise", Scientific Reports 5, Article number: 1108, doi:10.1038/srep11083, 2015.

[5] D. Newborough, C. Blomquist and P.A. Lepper, "The new generation of Electronic Click Detectors (ECD's): The development and field trial performance". Proc. Institute of Acoustics, Underwater Bio-Sonar and Bioacoustics, Vol. 23, Pt. 4, pp. 187-198, ISSN: 1-901656-37-3, July 2001, pp. 187-198.

[6] P.A. Lepper, N. Dumortier, K. Dudzinski and S. Datta, "Separation of Complex Echolocation Signal "Trains" from Multiple Bio-Sonar Sources", Proceedings of the 1st International Conference on Underwater Acoustic Measurements: Technologies and Results, 2, John Papadakis/Leit Bjorno, Heraklion, Greece, , ISBN 960-88702-08, 2005, pp. 913-918.

[7] V.L.G, Todd, W.D., Pearse, N.C., Tregenza, N.C., P.A., Lepper and I.B. Todd, "Diel echolocation activity of harbour porpoises (Phocoena phocoena) around North Sea offshore gas installations", ICES Journal of Marine Science Installations, 66, ISSN 101093/ICESJMS, 2009, pp. 112.

[8] M. Daehne, A. Gilles, K. Lucke, V. Peschko, S. Adler, K. Krugel, J. Sundermeyer and U. Siebert. "Effects of pile-driving on harbour porpoises (Phocoena phocoena) at the first offshore wind farm in Germany." Environ. Res. Lett. 8025002 doi:10.1088/17489326/8/2/025002, 2013. 